\title{
Article \\ Genetic Labeling of Cells Allows Identification and Tracking of Transgenic Platelets in Mice
}

\author{
Irena Krüger $\mathbb{D}^{D}$, Friedrich Reusswig, Kim Jürgen Krott, Celina Fabienne Lersch, Martina Spelleken \\ and Margitta Elvers*
}

Citation: Krüger, I.; Reusswig, F.; Krott, K.J.; Lersch, C.F.; Spelleken, M.; Elvers, M. Genetic Labeling of Cells Allows Identification and Tracking of Transgenic Platelets in Mice. Int. J. Mol. Sci. 2021, 22, 3710. https:// doi.org/10.3390/ijms22073710

Academic Editor:

Ana Garcia-Redondo

Received: 9 March 2021

Accepted: 29 March 2021

Published: 2 April 2021

Publisher's Note: MDPI stays neutral with regard to jurisdictional claims in published maps and institutional affiliations.

Copyright: (c) 2021 by the authors. Licensee MDPI, Basel, Switzerland. This article is an open access article distributed under the terms and conditions of the Creative Commons Attribution (CC BY) license (https:/ / creativecommons.org/licenses/by/ $4.0 /)$.
Department of Vascular and Endovascular Surgery, Experimental Vascular Medicine, Heinrich-Heine University Medical Center, 40225 Düsseldorf, Germany; Irena.Krueger@med.uni-duesseldorf.de (I.K.); FriedrichWilhelmBernd.Reusswig@med.uni-duesseldorf.de (F.R.); Kim-Juergen.Krott@med.uni-duesseldorf.de (K.J.K.); celer100@uni-duesseldorf.de (C.F.L.); Martina.Spelleken@med.uni-duesseldorf.de (M.S.)

* Correspondence: Margitta.Elvers@med.uni-duesseldorf.de

\begin{abstract}
Background: The use of knock-out mouse models is crucial to understand platelet activation and aggregation. Methods: Analysis of the global double fluorescent Cre reporter mouse $m T / m G$ that has been crossbred with the megakaryocyte/platelet specific PF4-Cre mouse. Results: Platelets show bright $m T$ (PF4-Cre negative) and $m G$ (PF4-Cre positive) fluorescence. However, a small proportion of leukocytes was positive for $m G$ fluorescence in PF4-Cre positive mice. In $m T / m G$;PF4-Cre mice, platelets, and megakaryocytes can be tracked by their specific fluorescence in blood smear, hematopoietic organs and upon thrombus formation. No differences in platelet activation and thrombus formation was observed between $m T / m G ; P F 4-C r e$ positive and negative mice. Furthermore, hemostasis and in vivo thrombus formation was comparable between genotypes as analyzed by intravital microscopy. Transplantation studies revealed that bone marrow of $m T / m G ; P F 4-C r e$ mice can be transferred to $C 57 B L / 6$ mice. Conclusions: The $m T / m G$ Cre reporter mouse is an appropriate model for real-time visualization of platelets, the analysis of cell morphology and the identification of nonrecombined platelets. Thus, $m T / m G ; P F 4$-Cre mice are important for the analysis of platelet-specific knockout mice. However, a small proportion of leukocytes exhibit $m G$ fluorescence. Therefore, the analysis of platelets beyond hemostasis and thrombosis should be critically evaluated when recombination of immune cells is increased.
\end{abstract}

Keywords: platelets; megakaryocytes; loxP; Cre recombinase; double fluorescent Cre reporter mouse; PF4-Cre

\section{Introduction}

Platelets are major players in hemostasis and thrombosis. At sites of vessel injury, platelets adhere, become activated, and form a hemostatic plug to maintain the integrity of the vessel wall after vascular damage. Under pathological conditions, platelet activation leads to thrombosis at sites of atherosclerotic plaque rupture that obstructs the blood flow in the circulation. Arterial thrombi are platelet rich and induce myocardial infarction or stroke [1-3].

Injury of the vessel and extravasation of blood from the circulation into the surrounding tissue initiates events at the site of vessel damage and in blood to seal the injury. Circulating platelets are recruited to the site of injury by the interaction of platelet glycoprotein (GP) Ib and von Willebrand Factor (vWF) that is exposed on collagen of the extracellular matrix. This interaction is modulated by extracellular and intracellular platelet proteins such as reelin [4] and PLD1 [5] and captures platelets from the blood stream under high shear conditions, initiates low integrin $\alpha_{\mathrm{IIb}} \beta_{3}$ activation and allows the binding of the collagen receptor GPVI to collagen exposed at the injured vessel [6,7]. Activation of GPVI induces platelet activation characterized by $\mathrm{Ca}^{2+}$ mobilization, release of the second wave 
mediators adenosine diphosphate (ADP) and thromboxane, stable platelet adhesion and aggregation by integrin $\alpha_{\mathrm{IIb}} \beta_{3}$ and is reinforced by reelin that is released from platelets upon GPVI stimulation [7-9]. Concomitantly, blood coagulation is initiated by tissue factor leading to the generation of thrombin. Fibrinogen is cleaved by thrombin to fibrin monomers that polymerize and are cross-linked by the transglutaminase factor XIIIa to stabilize the growing thrombus [10]. The ability of platelets to provide a pro-coagulant surface to allow the assembly of the coagulation complex and the property of thrombin to activate platelets via protease-activated receptors indicates a crosstalk of platelet-mediated primary hemostasis and the coagulation cascade (secondary hemostasis).

In the last decades, new technologies including intravital video microscopy have been developed to analyze thrombus formation in a living mouse. Different mouse models have been established to explore the role of platelets, blood coagulation proteins, endothelium, and the vessel wall during thrombus formation in mice that offers a new understanding of the physiology and the pathology in complex biological systems. However, the investigation of single genes/proteins and their impact in platelet activation and thrombus formation is impeded by the fact that platelets are anucleate cells, which do not allow analysis by classical molecular biological means. In the last decades, animal models for in vivo generation of genetically modified platelets have been established to investigate these genes and their role in platelet activation and thrombus formation. The generation of (platelet-specific) knockout mice and the transplantation of genetically modified human or murine platelet progenitor cells in myelo-conditioned mice have been helpful in the understanding of new pathological pathways and the development of anti-thrombotic drugs [11]. The generation of platelet-specific knockout mice with the Cre-loxP method and a megakaryocyte/platelet-specific promoter has been shown to exhibit an advantage compared to constitutive knockout mice with a deletion of the target gene in all cells of the organism. This is due to the fact that the phenotype of these mice can be directed to megakaryocytes/platelets and is not affected by other cells. Furthermore, lethal phenotypes as observed, e.g., for talin, filamin A, or CLEC-2 are a limiting factor of the classical approach of whole organism knockout mice [12-14]. Thus, the use of inducible or cell type-specific promoters in combination with the Cre-loxP knockout technique allows spatiotemporal knockout or targeted expression in a particular cell type without the developmental effects or effects of gene knockdown in non-target cell types.

However, the generation of platelet-specific knockout mice is time-consuming and there are problems arising from these mouse models. A general problem is the existence of so-called endogenous pseudo-loxP sites, which can be targeted by Cre as well [15]. Furthermore, promoters that are only active during late stages of megakaryopoiesis such as the platelet factor 4 (PF4) are not an appropriate model to study megakaryopoiesis [16,17]. The specificity of some of the promoters are not always fully restricted to one cell type as demonstrated by the erythrocyte/megakaryocyte-specific GATA-1 promoter [18]. The promoter that drives the expression of the Cre recombinase has to be strong enough to gather complete DNA recombination important to generate a complete knockout of the target gene and to avoid the generation of mosaic animals. The PF4 which is specific for the megakaryocytic lineage is nowadays used in many thrombosis studies with knockout mice and mice are born with a platelet-specific deletion of the target gene [19]. However, knockout efficiency of these conditional knockout mice is less than $95 \%$, and thus wildtype platelets are still in the circulation of PF4-Cre mice and cannot be identified properly [19].

In this study, we were able to show that the global double fluorescent Cre reporter mouse $m T / m G$ that has been crossbred with the PF4-Cre mouse is an efficient elegant model for megakaryocyte/platelet tracing, the analysis of cell morphology, transplantation studies and the differentiation of knockout versus wildtype platelets in these mice. 


\section{Results}

\subsection{Main Text}

\subsubsection{Autofluorescence of Platelets in $m T / m G ; P F 4-C r e$ transgenic Mice}

The double-fluorescent Cre reporter mouse $m T / m G$ has been crossbred with the megakaryocyte/platelet specific PF4-Cre mouse (Figure 1A). The $m T / m G$ mouse expresses membranetargeted tandem dimer Tomato $(m T)$ prior to Cre-mediated excision and membrane-targeted green fluorescent protein $(m G)$ after excision. As a result, $m T / m G$;PF4-Cre mice exhibit green fluorescent platelets in the circulation while platelets isolated from PF4-Cre negative mice show red fluorescence (Figure 1B). Fluorescence intensity of platelets can be observed already in heterozygous mice and is increased in the homozygous genetic background (Figure 1B). Fluorescence signals were detected in the fluorescein-5-isothiocyanate (FITC) (EGFP, $m G$ fluorescence) and the phycoerythrin (PE) (Tomato, $m T$ fluorescence) channels using $m T / m G$;PF4-Cre positive and negative heterozygous and homozygous mice. To confirm cell specificity of genetic labeling of blood cells in $m T / m G$ mice, platelets, red blood cells and leukocytes were analyzed for their fluorescence characteristics by flow cytometry. The analysis of $m G$ positive cells revealed no fluorescence of red blood cells in PF4-Cre negative and positive mice but strong $m G$ fluorescence $(99.46 \%$ ) of platelets in PF4-Cre positive but not in PF4-Cre negative mice. However, we detected around $13 \%$ of $m G$ positive leukocytes in PF4-Cre positive mice (Figure 1C). We next analyzed the number of $m T$ positive blood cells and detected almost no fluorescence of red blood cells, neither in PF4-Cre negative nor positive mice (Figure 1D). As predicted, platelets from PF4-Cre negative mice exhibit strong $m T$ fluorescence (92.92\%) while platelets from $P F 4$-Cre positive mice were negative for $m T$ fluorescence. In contrast, strong $m T$ fluorescence of leukocytes of both, $P F 4$-Cre negative and positive mice, was detected (Figure 1D). Blood cells from C57BL/6 mice were used as controls and show no $m G$ and $m T$ fluorescence as expected (Figure 1C,D). Fluorescence activated cell sorting of $m T$ and $m G$-labeled blood cells was confirmed by their forward/sideward scatter profiles and cell type specific labeling of leukocytes (CD45), platelets (GPIb), and red blood cells (TER119) (Figure 1E). Taken together, nearly all leukocytes and platelets expressed a marker gene, either $m T$ or $m G$ while red blood cells exhibit only very low fluorescence signals. Although it is described that the PF4-Cre recombinase is restricted to the megakaryocyte/platelet lineage, a small population of leukocytes $(13.39 \%)$ expressed $m G$ while a large population of leukocytes $(64.89 \%)$ express $m T$.

\subsubsection{Fluorescently labeled Platelets and Megakaryocytes in Blood Smear, Spleen, and} Bone Marrow of Transgenic Mice

We next investigated $m T$ and $m G$ labeling in the blood smear, spleen, and bone marrow of $m T / m G ; P F 4$-Cre mice (Figure 2). Examination of the blood smear revealed strong $m G$ fluorescence of platelets as shown by fluorescence microscopy (Figure 2A,B) using blood from PF4-Cre positive mice. $m T$ fluorescence of platelets and leukocytes was detected only in PF4-Cre negative mice. In contrast, DIC/brightfield images provided strong evidence that red blood cells exhibit almost no fluorescence, neither $m T$ nor $m G$ (Figure 2A) suggesting that the $m T / m G ; P F 4$-Cre reporter mouse is not appropriate for genetic labeling of red blood cells. 
A

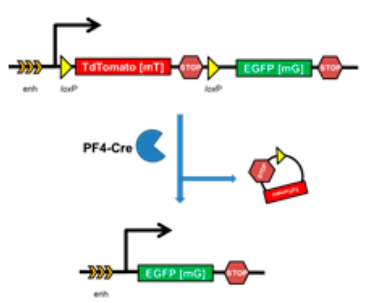

C

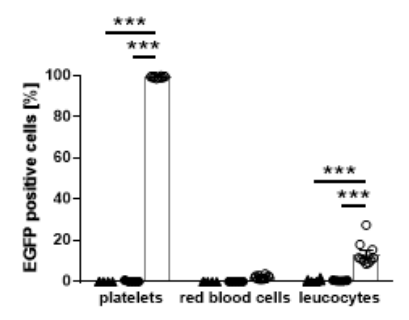

E

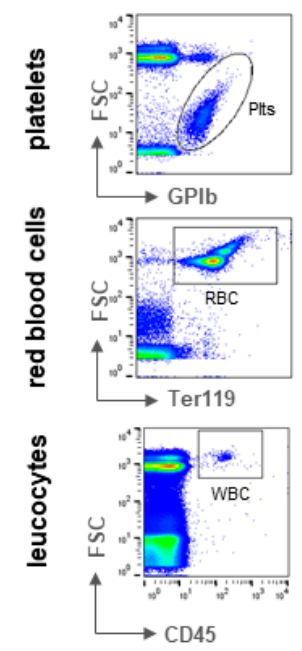

B

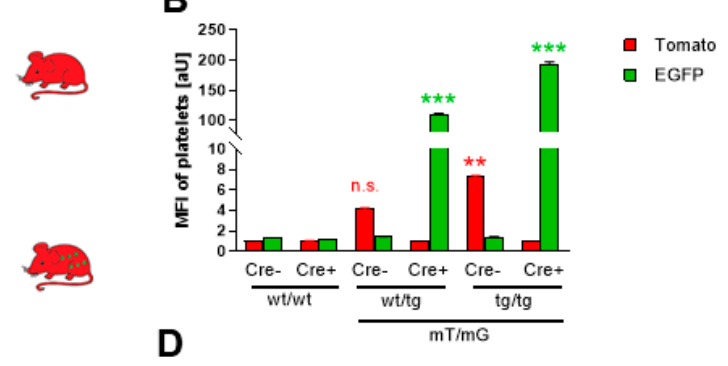

D

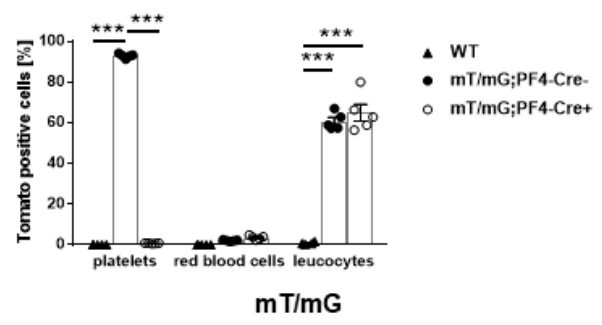

wildtype PF4-Cre- PF4-Cre+
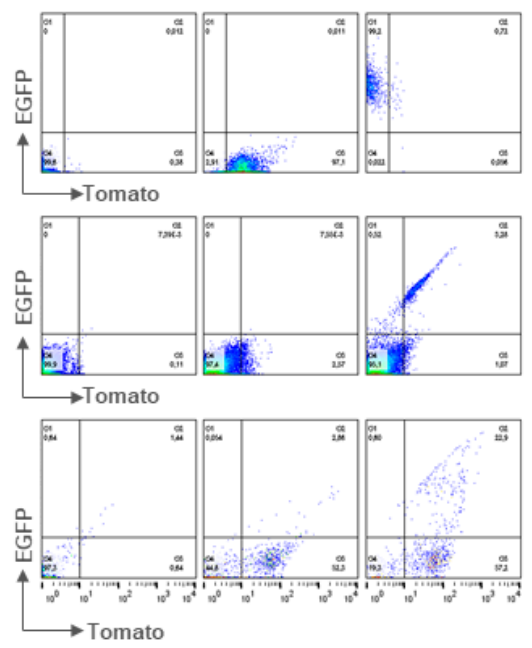

Figure 1. $m T$ and $m G$ fluorescence of blood cells in $m T / m G$; PF4-Cre reporter mice. (A) Schematic diagram of the genetic construct of $m T / m G$; PF4-Cre mice. The $m T / m G$ construct consists of an enhancer (enh.) and a promoter (black arrow) region driving a loxP-flanked coding sequence of membranetargeted tandem dimer Tomato $(m T)$ resulting in tdTomato expression with membrane localization in every tissue. A stop codon inhibits the gene expression of membrane-targeted green fluorescent protein $(m G)$. After Cre-mediated intrachromosomal recombination, the $m T$ sequence is excised allowing the promoter/enhancer to drive the expression of $m G$ in platelets and megakaryocytes. Yellow triangles represent loxP target sites for Cre-mediated recombination. (B) Mean fluorescence intensity (MFI) of $m T$ and $m G$ fluorescence in platelets regarding their specific side (SSC) and forward scatter (FSC) profile as determined by flow cytometry using whole blood of wildtype, heterozygous and homozygous $m T / m G$ PF4-Cre negative and positive mice, $\mathrm{n}=3-6$. (C-E) Cell specific expression of $m T$ or $m G$ fluorescence was determined in platelets (DyLight649-labeled anti-mouse GPIb $\alpha$ ), red blood cells (APC-labeled rat anti-mouse TER-119) and leucocytes (APC-labeled rat anti-mouse CD45). Fluorescence signals were detected in the FITC $(m G)$ and PE $(m T)$ channels. (C,D) MFI of $m G$ (C) and $m T$ (D) fluorescent cell populations in washed whole blood of WT and $m T / m G$;PF4-Cre negative and positive mice using flow cytometry, $n=5$. (E) Representative dot blots showing the gating strategy for each cell population. The MFI of $m G$ and $m T$ fluorescence of different blood cells isolated from $m T / m G ; P F 4-C r e$ mice. Statistical analysis was performed by Two-way Anova Sidak's post hoc test $(\mathbf{A}, \mathbf{B})$ and Two-way Anova Tukey's post hoc test $(\mathbf{C}, \mathbf{D})$. Bar graphs depict mean values \pm SEM, ${ }^{*} p<0.05 ;{ }^{* *} p<0.01$ and ${ }^{* * *} p<0.001$. 

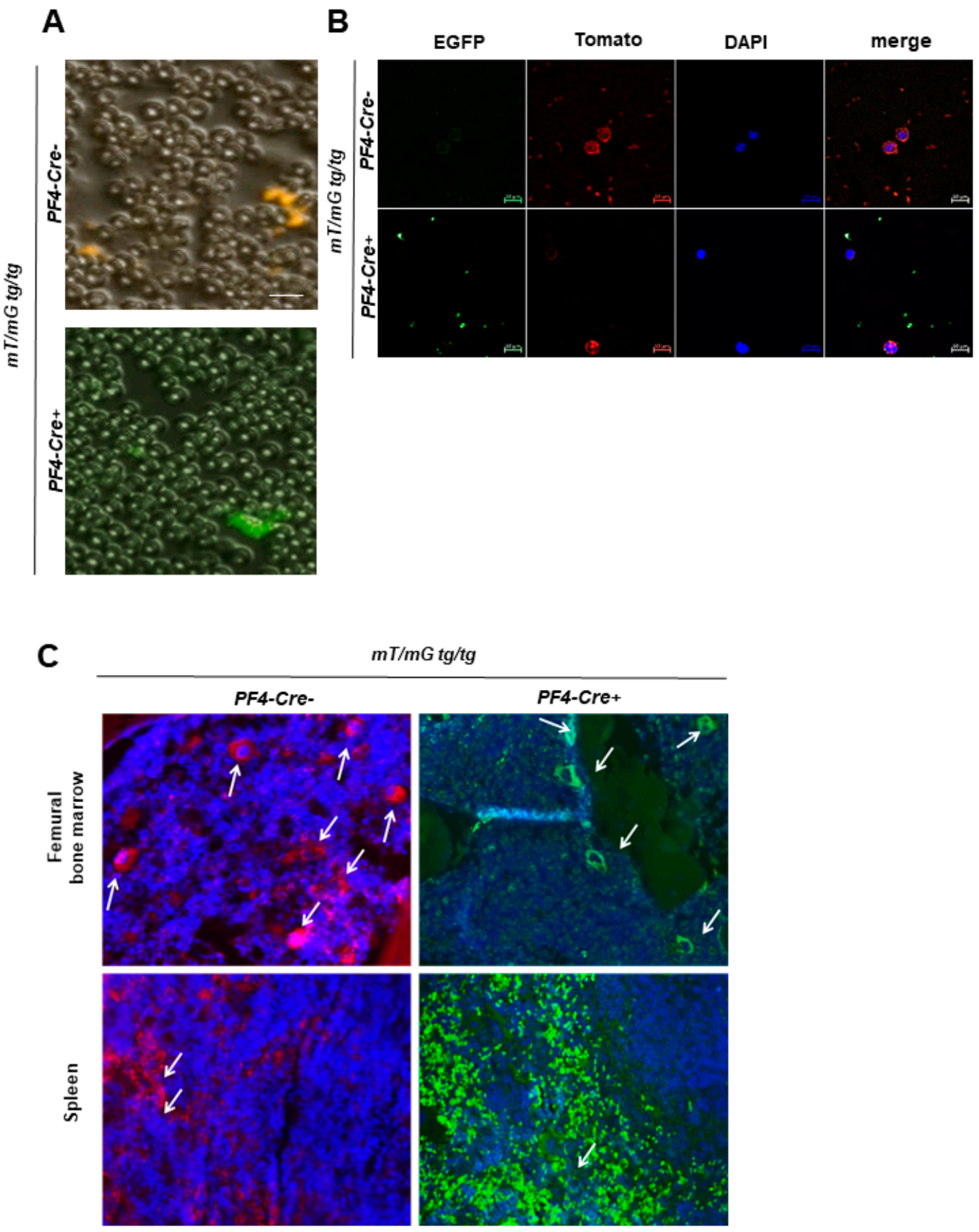

Figure 2. $\mathrm{mT}$ and $\mathrm{mG}$ fluorescence of platelets and megakaryocytes in blood smear, spleen and femur of mT/mG; PF4Cre transgenic mice. (A,B) Blood smear samples of $\mathrm{mT} / \mathrm{mG}$ PF4-Cre negative and positive mice were analyzed for $\mathrm{mT}$ and $\mathrm{mG}$ fluorescence of blood cells. (A) Representative images of $\mathrm{mT}$ (upper panel) and mG (lower panel) fluorescence of platelets in blood smear of transgenic mice using differential interference contrast (DIC), $n=8-10$, scale bar $20 \mu \mathrm{m}$. (B) Representative images of single channels are shown representing either $\mathrm{mT}$ or $\mathrm{mG}$ fluorescence. Additionally, DAPI staining was performed to identify nucleus containing cells such as leukocytes in blood smear, $n=8-10$, scale bar $10 \mu \mathrm{m}$. (C) Analysis of hematopoietic active tissue (spleen and femoral bone marrow) in $\mathrm{mT} / \mathrm{mG} ; \mathrm{PF} 4-\mathrm{Cre}$ mice. Representative images of $\mathrm{mT}$ fluorescence (mT/mG;PF4-Cre negative) and $\mathrm{mG}$ fluorescence (mT/mG;PF4-Cre positive). DAPI was used to stain nuclei in spleen and femur. White arrows indicate megakaryocytes. $n=3$. 
To test the $m T / m G$ reporter mouse for lineage-specific cell appearance and morphology, we analyzed $m T$ and $m G$ labeling in $m T / m G ; P F 4$-Cre mice in different hematopoietic tissues such as spleen and bone marrow. An array of megakaryocytes can be identified by the fluorescence markers. In detail, $m T$ positive megakaryocytes were identified in the bone marrow of the femur and in low numbers in the spleen of PF4-Cre negative mice. Accordingly, $m G$ positive megakaryocytes were detected in both organs of PF4-Cre positive mice. Moreover, we identified a large number of platelets in the spleen that exhibit either $m T$ (PF4-Cre negative mice) or $m G$ fluorescence (PF4-Cre positive mice) (Figure 2C).

2.1.3. Unaltered Platelet Activation and Receptor Surface Expression in PF4-Cre negative and PF4-Cre positive Mice

To test if platelet numbers and characteristics differ in $m T / m G$;PF4-Cre positive and negative mice, we determined different platelet parameters. First, we analyzed platelet counts and size and the exposure of platelet glycoproteins in these mice and found no significant differences (Figure 3A,C). To investigate platelets from different genotypes in more detail, we investigated platelet adhesion on fibrinogen at different time points and determined platelet activation. First, platelet adhesion to immobilized fibrinogen after 5, 20, and $60 \mathrm{~min}$ revealed no differences between genetically different experimental groups (Figure 3D,E). However, we detected individual platelets with $m T$ fluorescence in $m T / m G ; P F 4$-Cre positive mice (Figure 3E, lower panel) suggesting the existence of single wildtype platelets in these mice.

To analyze platelet activation, P-selectin exposure as marker for degranulation and fibrinogen binding to integrin $\alpha_{\mathrm{IIb}} \beta_{3}$ (fibrinogen receptor) as marker for integrin activation was determined by flow cytometry (Figure 3F,G). The activation of platelets with different agonists that induce signal transduction via G-protein coupled receptors or collagen receptor activation showed no major differences between platelets isolated from $m T / m G ; P F 4-C r e$ positive and negative mice (Figure 3F,G). Taken together, no major differences in platelet characteristics and platelet activation were found between different groups and non-recombined platelets can be identified by $m T$ fluorescence suggesting no Cre recombinase activity in the respective precursor cell.

\subsubsection{Comparable Thrombus Formation in PF4-Cre negative and positive $m T / m G$ transgenic Mice}

To analyze platelet activation under more physiological conditions, platelet adhesion and aggregate formation leading to three-dimensional thrombus formation under flow conditions were investigated ex vivo. To this end, cover slips were coated with $200 \mu \mathrm{g} / \mathrm{mL}$ collagen and whole blood from $\mathrm{mT} / \mathrm{mG}$;PF4-Cre positive and negative mice was perfused through the chamber at an arterial shear rate of $1000 \mathrm{~s}^{-1}$ (Figure 4A). As shown in Figure $4 \mathrm{~A}$ and B, no differences in the formation of three-dimensional thrombi were observed between different groups. Whole blood from PF4-Cre negative mice showed strong $m T$ fluorescence of platelets within the thrombus. Accordingly, the perfusion of whole blood from PF4-Cre positive mice resulted in strong $m G$ fluorescence of the thrombus because the thrombi consist mainly of platelets and only single leukocytes and red blood cells can be found in the thrombus [20] (Figure 4A,B). 
A

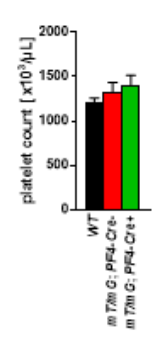

E

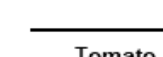

B

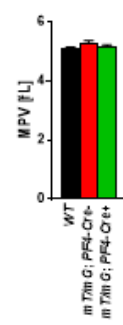

$m T / m G ;$ PF4-Cre-

eGFP
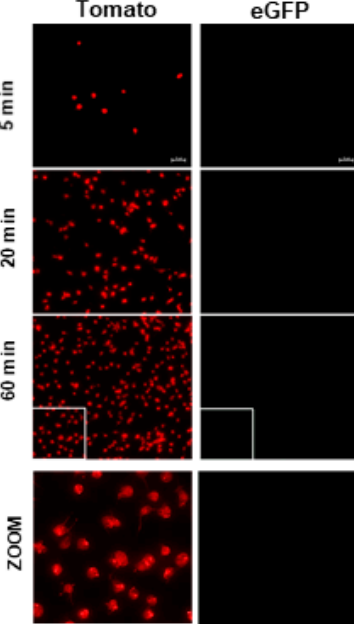

$\mathbf{F}$

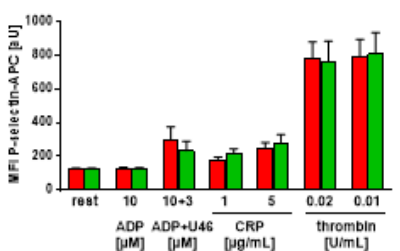

C

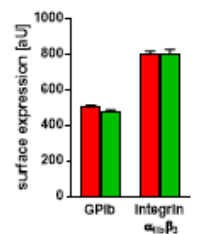

D

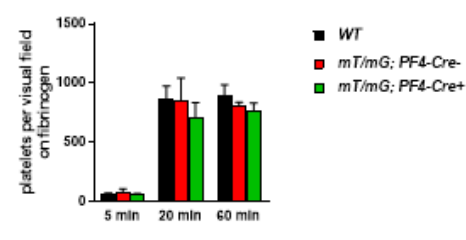

$m T / m G ;$ PF4-Cre+

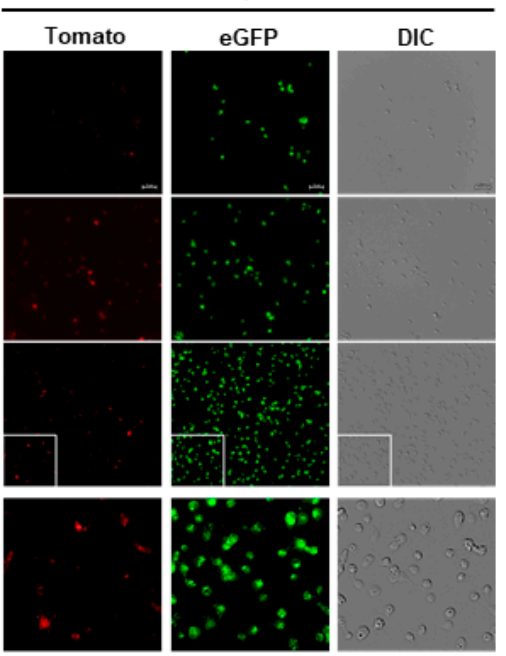

G

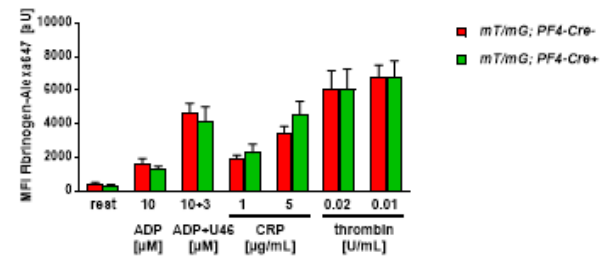

Figure 3. Unaltered platelet activation and receptor surface expression in $m T / m G$ PF4-Cre negative and positive mice. (A) No differences were observed in platelet counts, (B) platelet size (mean platelet volume, MPV) between $W T$ and $m T / m G ; P F 4-C r e$ negative and positive mice and (C) in the expression of glycoprotein (GP) Ib and integrin $\alpha_{\mathrm{IIb}} \beta_{3}$ between $m T / m G ; P F 4$-Cre negative and positive mice. GP expression was determined using DyLight649-labeled anti-mouse GPIb $\alpha$ and DyLight649labeled anti-mouse integrin $\alpha_{\mathrm{IIb}} \beta_{3}$ using flow cytometry. Statistical analysis was performed by Kruskal-Wallis test and Dunn's post hoc test $(\mathbf{A}, \mathbf{B})$ and two-tailed Student's t-test $(\mathbf{C})$. Bar graphs depict mean values \pm SEM, $n=6-8$. (D,E) Isolated platelets were allowed to adhere to fibrinogen [100 $\mu \mathrm{g} / \mathrm{mL}$ ] for indicated time points and fixed with PFA. Statistical analysis was performed by two-Way ANOVA Tukey's post hoc test. Bar graphs depict mean values \pm SEM, $n=3-4$. (D) The number of adherent platelets per visual field was determined. (E) Representative images showing platelet adhesion and platelet morphology of $m T$ and $m G$ fluorescent platelets, $n=3-4$, scale bar $10 \mu \mathrm{m}$. (F,G) Platelet degranulation (P-selectin exposure) and fibrinogen binding (marker for integrin activation) following activation with indicated agonists was determined in washed whole blood using flow cytometry. (F) P-selectin externalization was analyzed with an anti-human/mouse CD62PAPC antibody, $n=6-7$. (G) Fibrinogen binding was determined using fibrinogen-Alexa Fluor ${ }^{\mathrm{TM}}$ 647, $n=5-6$. Statistical analysis was performed by two-tailed Student's $t$-test. Bar graphs depict mean values $\pm \mathrm{SEM}$. CRP = collagen-related peptide, $\mathrm{U} 46=$ Thromboxane $\mathrm{A} 2$ analogue, $\mathrm{ADP}=$ adenosine diphosphate, $\mathrm{DIC}=$ differential interference contrast. 
A
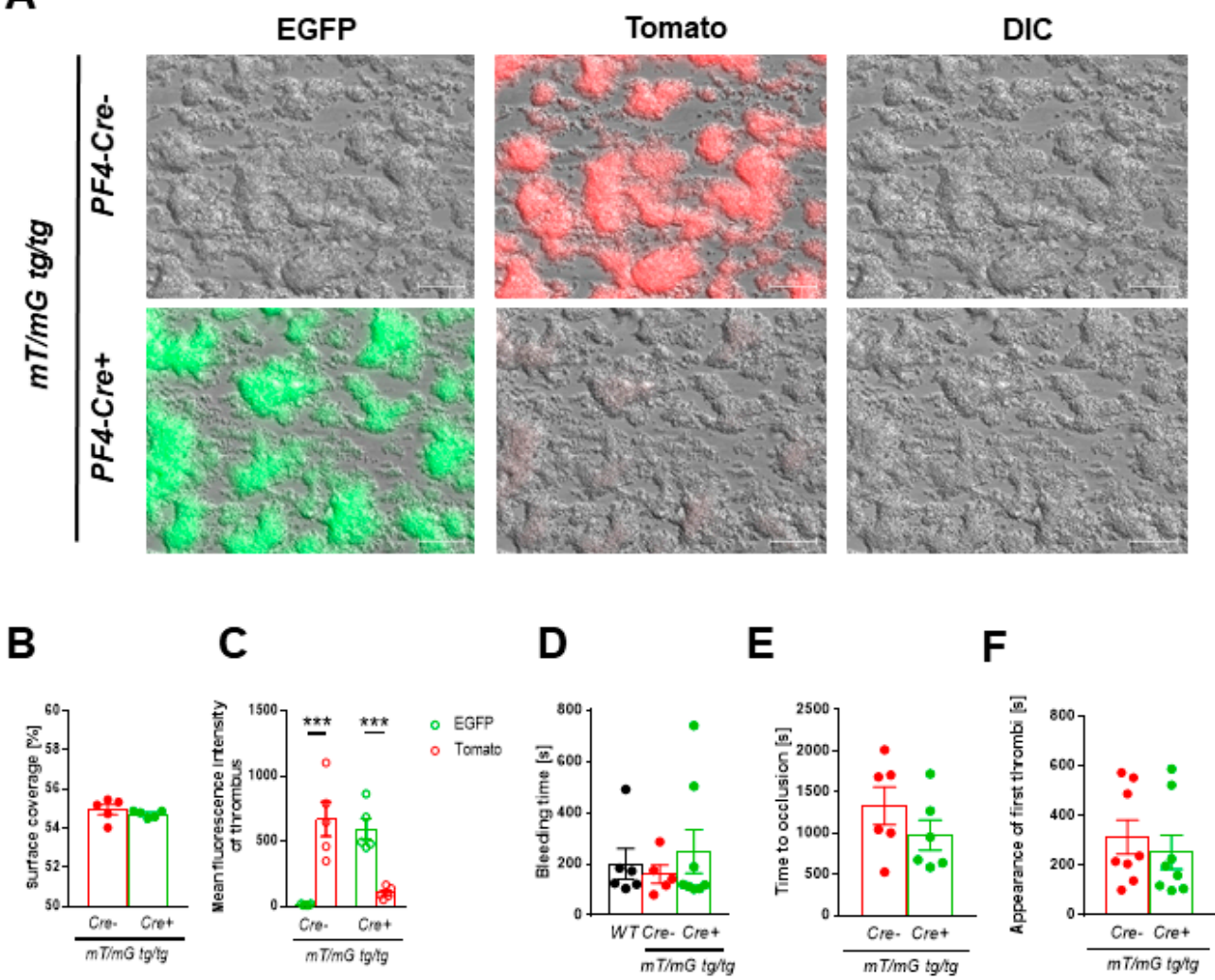

\section{G}

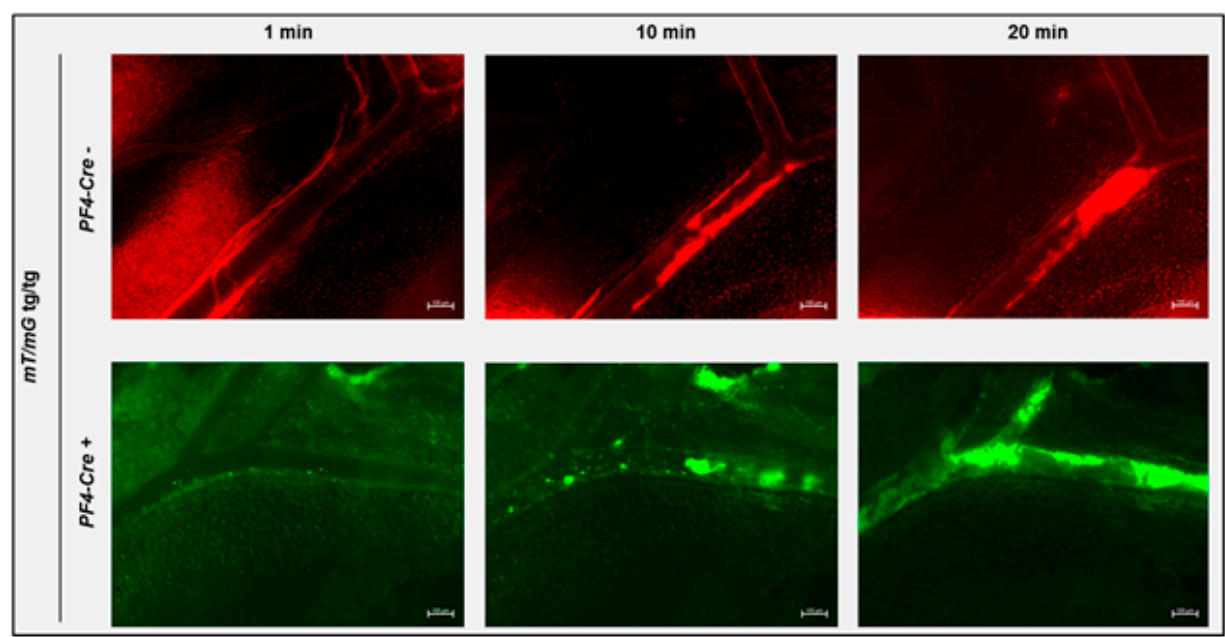

Figure 4. Unaltered thrombus formation in $m T / m G ; P F 4-C r e$ negative and positive mice ex vivo and in vivo. (A-C) Murine whole blood was perfused over a collagen-coated matrix $(200 \mu \mathrm{g} / \mathrm{mL})$ with a shear rate of $1000 \mathrm{~s}^{-1}$. (A) Representative images of thrombus formation showing $m T$ (upper panel) or $m G$ (lower panel) fluorescence of platelets that have been incorporated into the growing thrombus. Determination of (B) surface coverage and (C) Mean fluorescence intensity (MFI) of thrombi to quantify thrombus formation of each genotype, $n=5$, scale bar $50 \mu \mathrm{m}$. (D) Hemostasis was analyzed via tail bleeding time of $m T / m G ; P F 4-C r e$ negative and positive mice. No differences between genotypes could be detected, $n=5-7$. (E-G) In vivo thrombus formation in mesenteric arteries after $\mathrm{FeCl}_{3}$ induced injury was analyzed using intravital microscopy. (E) Time to occlusion of the injured vessel and (F) appearance of first thrombi were determined. (G) Platelet aggregate formation at indicated time point was monitored by $m T$ and $m G$ fluorescence of platelets. $m T$ and $m G$ fluorescence of platelets was stable and allowed visualization of thrombus growth until full occlusion of the vessel in $m T / m G$;PF4-Cre mice, $n=6-8$, scale bar $100 \mu \mathrm{m}$. Statistical analysis was performed by two-tailed Student's $t$-test $(\mathbf{B}, \mathbf{C}, \mathbf{E}, \mathbf{F})$ and Kruskal-Wallis test followed by Dunn's post hoc test (D). Bar graphs depict mean values \pm SEM, ${ }^{*} p<0.05,{ }^{* *} p<0.01$ and ${ }^{* * *} p<0.001$. 


\subsubsection{Tracking of $\mathrm{mT} / \mathrm{mG}$ positive Platelets via Intravital Microscopy}

Next, we investigated hemostasis in $m T / m G ; P F 4-C r e$ mice. In this model, the ability of mice to arrest bleeding after cutting the tail tip with a scalpel to induce a defined tail wound serves as an indicator for normal blood clotting. In line with the ex vivo results shown above, no differences in hemostasis were observed when the time until bleeding stops was determined (Figure 4C).

To analyze the formation of arterial thrombi in vivo, $m T / m G$;PF4-Cre mice were investigated by intravital microscopy (Figure 4D-F). We injured mesenteric arterioles by topical application of $\mathrm{FeCl}_{3}$ and tracked $m T$ and $m G$ fluorescent platelets in vivo. As shown in Figure 4D-F, platelets showed bright fluorescence of either $m T$ in $m T / m G ; P F 4$-Cre negative mice or $m G$ in $m T / m G ; P F 4-C r e$ positive mice. Furthermore, no differences were observed when we determined the appearance of first thrombi at the site of injury and the time to full occlusion of the vessel in these mice suggesting no major differences in arterial thrombosis in vivo (Figure $4 \mathrm{D}-\mathrm{F}$ ).

Taken together, platelet aggregates and thrombus formation can be tracked by intravital microscopy and reveals unaltered platelet adhesion and thrombus formation in the double fluorescent cre reporter mouse.

\subsubsection{Transfer of Bone Marrow from $m T / m G$;PF4-Cre positive Mice to C57BL/6 Mice}

The first characterization of $m T / m G$ mice suggested that these mice can be used for transplantation studies [21]. Therefore, we wanted to know if the transfer of bone marrow from $m T / m G ; P F 4-C r e$ positive mice to $C 57 B L / 6$ mice provides the advantage for platelet tracing and the analysis of platelet morphology in bone marrow chimeric mice. Bone marrow of $m T / m G ; P F 4-C r e$ positive mice was extracted and transferred into C57BL/6 mice. Successful transfer of bone marrow was monitored by determination of platelet counts (Figure 5A), the number of red blood cells (Figure 5B) and leukocytes (Figure 5C), hemoglobin plasma levels (Figure 5D), and mean platelet volume (platelet size) (Figure 5E). When C57BL/6 mice were reconstituted with $m T / m G ; P F 4$-Cre bone marrow we were able to detect $m G$ positive platelets in these mice but not in control C57BL/6 mice using flow cytometry (Figure 5F). In summary, the transfer of bone marrow from $m T / m G ; P F 4$-Cre positive mice to C57BL/6 mice led to the appearance of $m G$-positive platelets in the circulation of chimeric mice.

A

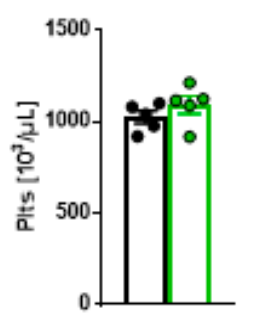

B

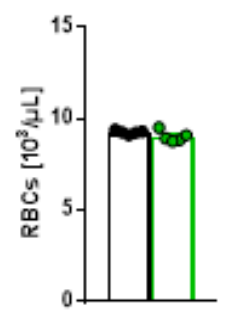

C

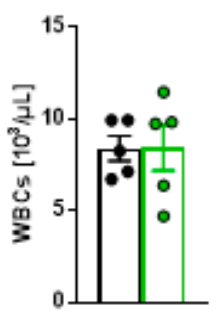

D

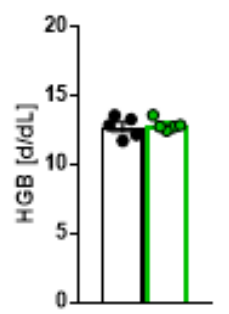

$\mathbf{E}$

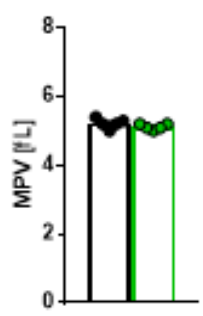

- C57BL/6J

C57BL/6J chimera

donor: $\mathrm{mT} / \mathrm{mG}$; PF4-Cre+

Figure 5. Cont. 

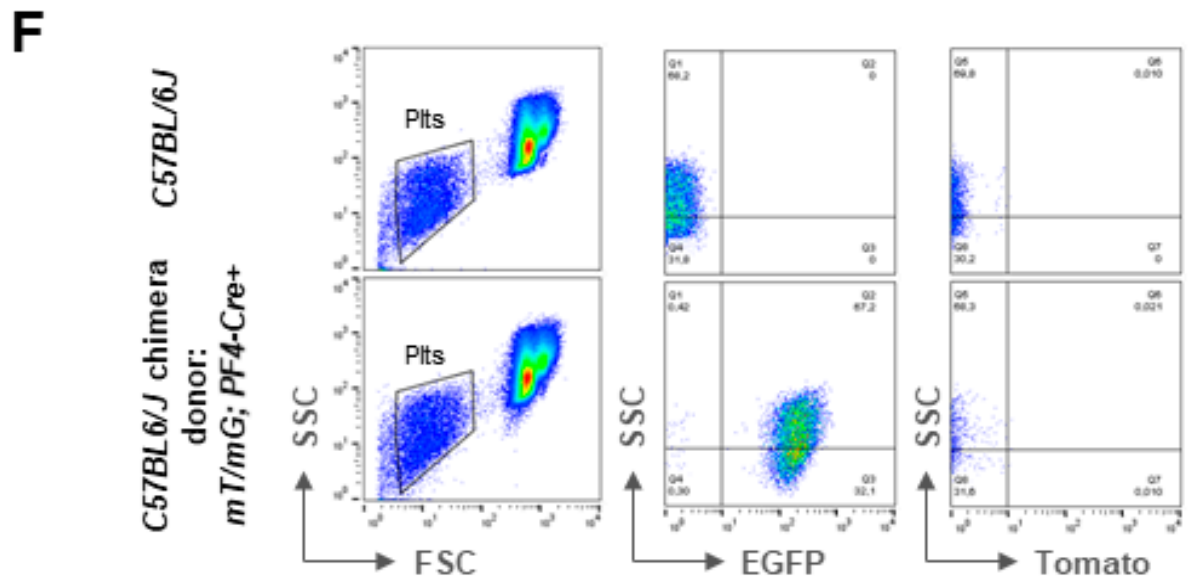

Figure 5. Transfer of bone marrow from $m T / m G$; PF4-Cre positive mice to C57BL/6 mice leads to the appearance of $m G$-positive platelets in the circulation of chimeric mice. C57BL/6 mice were exposed to sublethal irradiation and received bone marrow cells of $m T / m G ; P F 4-C r e$ positive mice. Platelet count (A), number of red blood cells (B) and leukocytes (C), hemoglobin concentration (D) and platelet size (E, mean platelet volume) were determined in heparinized whole blood by a hematology analyzer (Sysmex, Norderstedt, Germany). No major differences between bone marrow chimeric mice and C57BL/6 controls could be detected, $n=5$. (F) Platelets from bone marrow chimeric mice were analyzed regarding their $m T$ and $m G$ fluorescence in the SSC and FSC specific profile. Only platelets with $m G$ fluorescence could be detected, $n=5$. Statistical analysis was performed by two-tailed Student's t-test. Bar graphs depict mean values \pm SEM. Plts = platelets.

\section{Discussion}

The present study revealed that the double fluorescent Cre reporter mouse that expresses dtTomato prior to excision and EGFP following excision is a useful tool for tracing megakaryocytes and platelets and visualizing thrombus formation in real time by intravital microscopy. Platelets and megakaryocytes from $m T / m G ; P F 4-C r e$ positive mice showed bright green fluorescence in the circulation (platelets) and in tissue such as the spleen (platelets and megakaryocytes) or bone marrow (megakaryocytes). Since there is no complete knockout efficiency in conditional knockout mice, the $m T / m G ; P F 4-C r e$ mouse represents an important tool for using the Cre/loxP system in mice to distinguish between wildtype and knock-out platelets because platelets with $m T$ fluorescence can be identified in this mouse line representing non-recombined platelets without Cre recombinase activity in the progenitor cell. No differences in platelet activation and thrombus formation have been observed between $m T / m G ; P F 4$-Cre positive and negative platelets and mice. Furthermore, we show that platelet count and size, platelet adhesion and bleeding times of $m T / m G ; P F 4-C r e$ transgenic mice are comparable to wildtype controls or already published data from our group (flow chamber, injury of mesenteric arterioles) [20,21]. Thus, mating these mice with a conditional knockout mouse where the gene knockout is only in Cre-expressing cells ensures that the observed phenotype in these mice can be dedicated to the loss of a specific gene in megakaryocytes. Furthermore, the $m T / m G ; P F 4-C r e$ mouse can be used for transplantation studies because the transplantation of bone marrow from $m T / m G ; P F 4-C r e$ positive mice to $C 57 \mathrm{BL} / 6$ mice allowed tracing of platelets in the circulation of bone marrow chimera.

The double fluorescent $m T / m G$ mouse line provides several advantages for the analysis of platelet activation and thrombus formation. First, PF4-Cre genotyping of these mice is simple in an $m T / m G$ homozygous background because platelets can be easily detected by flow cytometry using either the EGFP ( $m G$ fluorescence) or the Tomato channel ( $m T$ fluorescence) to distinguish between PF4-Cre positive and negative mice. Furthermore, the reporter mouse allows real-time visualization of platelets of both recombined and nonrecombined cells at single cell resolution (Figure 4F) in vivo. The bleed-through between the fluorescent channels is minimal as already observed by Muzumdar and colleagues and both, $m T$ and $m G$ are photostable [22]. The absolute brightness and photostability of 
$m G$ and $m T$ fluorescence was also observed in $m T / m G ; P F 4$-Cre positive and negative mice allowing the tracing of platelets after vascular injury of mesenteric arterioles (Figure 4D-F).

The results of the present study clearly shows bright fluorescence of platelets either with $m T$ (PF4-Cre negative mice) or with $m G$ (PF4-Cre positive mice) in vitro and in vivo. However, flow cytometric analysis revealed almost no fluorescence of red blood cells, neither in PF4-Cre negative nor in PF4-Cre positive mice. This might be due to the fact, that the major difficulty of fluorescent imaging of proteins, cells, and tissues within whole animals is the light absorption by hemoglobin, as well as light scattering [23,24]. Both, absorption and scattering become less distinct as the light wavelength increases. The "optical window", which is most transparent for the visualization in living tissues, is considered to be between 650-700 and $1100 \mathrm{~nm}$ suggesting the development of bright far-red or near infrared fluorescent proteins to increase sensitivity of whole body imaging techniques. However, the wavelength for tdTomato is 554/581 nm (excitation/emission) and for EGFP 488/509 nm (excitation/emission). This might explain the almost absent fluorescence of red blood cells.

Furthermore, we detected a small proportion of $m G$ positive leukocytes (Figure 1C-E) raising concerns about the specificity of the PF4 promoter for megakaryocyte/plateletrestricted Cre expression. Different groups in the past already doubted about the specificity of the PF4 promoter $[25,26]$ and described a broader expression of the mouse PF4-Cre transgene beyond the megakaryocyte lineage. Calaminus and colleagues demonstrated that the activity of the PF4-Cre recombinase is not restricted to megakaryocytes and platelets but extends to other myeloid and lymphoid lineages at significant levels between 15 and $60 \%$ [25]. They show that PF4-Cre also recombines in both fetal liver and bone marrow hematopoietic stem cells (HSCs), including the most primitive fraction containing the long-term repopulating HSCs.

In line with $m G$ fluorescence of CD45 positive cells detected by flow cytometry, Pertuy and colleagues found a minor fraction of CD45-positive cells in circulating blood but also recombined cells of monocyte/macrophage origin in all tissues [26]. In contrast, a study by Rudolph and colleagues confirmed lineage specificity of PF4-Cre mice that have been crossed with conditional ADAP (adhesion and degranulation-promoting adapter protein) knockout mice [27]. They generated a reporter strain by crossing PF4-Cre mice to Rosa26$t d R F P$ (tdRFP, tandem-dimer red fluorescent protein) reporter mice and could not detect any effects of Cre recombinase in other cells beyond platelets and megakaryocytes [27]. Taken together, the use of PF4-Cre mice and the identification of $m G$ positive cells always should be examined carefully, especially under inflammatory conditions when recombination in immune cells is further increased.

The $m T / m G$ Cre reporter mouse expresses a membrane-targeted fluorescent protein. The aim to attach a membrane tag to the reporter protein was to aid in visualization of fine processes as observed in neurons [22]. Muzumdar and colleagues showed that membrane localization of a fluorescent marker is highly effective and probably superior to cytoplasmic localization to outline, e.g., cell morphology. The analysis of $m \mathrm{~m} / \mathrm{mG} ; \mathrm{PF} 4-\mathrm{Cre}$ mice revealed more cytoplasmic fluorescence of both, $m T$ and $m G$, in platelets (Figure 3E) that is highly efficient to label and trace platelets but membrane localization of $m T$ in leukocytes (Figure 2B). However, megakaryocytes of the bone marrow and spleen exhibit more membrane-localized fluorescence of $m T$ and $m G$ (Figure 2C) as already observed by Pertuy and colleagues [26] This might be due to the fact that platelets are anuclear small cells of megakaryocyte origin. Nevertheless, bright fluorescence and thus identification of platelets in $m T / m G ; P F 4-C r e$ positive and negative mice could be observed (Figure $4 \mathrm{~F}$ ) that allows tracing of platelets not only ex vivo but also in vivo to analyze arterial thrombosis without additional labeling of platelets by a fluorescent dye (Figure 4).

Taken together, the presented results indicate that the $m T / m G ; P F 4-C r e$ mouse line is an elegant tool for easy genotyping of mice, direct and real-time visualization of platelets in vitro and in vivo and the identification of non-recombined platelets. Thus, the double fluorescent PF4-Cre mice are important for the analysis of platelet-specific knockout mice to 
dedicate the observed phenotype of transgenic mice to the megakaryocyte/platelet lineage. However, lineage-specific tracing in these mice is leaky to a small extend because a small proportion ( 13\%) of CD45-positive cells exhibit $m G$ fluorescence. Therefore, the analysis of platelets and their role in different cellular processes or diseases beyond hemostasis and thrombosis has been critically performed, especially when inflammation plays a role and therefore recombination in immune cells is increased.

\section{Materials and Methods}

\subsection{Animals}

Reporter mice (Gt(ROSA)26Sor ${ }^{\text {tm4} 4(A C T B-t d T o m a t o,-E G F P) L u o / J, ~ s t o c k ~ n o . ~ 007576) ~ w e r e ~ p u r-~}$ chased from The Jackson Laboratory (Bar Harbor, Maine, USA). This double fluorescent Cre reporter mouse called $m T / m G$ reporter mouse expresses membrane-targeted tdTomato $\left({ }^{\prime \prime} m T^{\prime \prime}\right)$ prior to Cre excision and membrane-targeted EGFP $\left({ }^{\prime \prime} m G^{\prime \prime}\right)$ following Cre excision [22]. These mice were crossbred with PF4-Cre transgenic mice (The Jackson Laboratory, stock no. 008535, C57BL/6-Tg(Pf4-icre)Q3Rsko/J, Bar Harbor, Maine, USA) to confine the expression of EGFP to megakaryocytes and platelets [19]. The animals were bred in the animal facility of University Düsseldorf. As WT controls served either littermates, which had a wildtype genotype for $m T / m G$ and C57BL/6 mice, which were bought at Janvier Labs (Le Genest-Saint-Isle, France).

All animal experiments were performed under adherence of the EU Directive 2010/63/EU and German animal welfare act using a protocol approved by the Heinrich-Heine-University Animal Care Committee and he district government of North Rhine-Westphalia (LANUV NRW, permit numbers: 84-02.05.20.12.284, 84-02.05.40.16.073, 84-02.04.2013.A210 and 84 02.04.2016.A493).

\subsection{Chemicals}

Platelets were activated with ADP (Sigma-Aldrich, St. Louis, MO, USA), thrombin (Roche, Basel, Switzerland), CRP (Richard Farndale, University of Cambridge, United Kingdom) and U46619 (Enzo Life Sciences, Lörrach, Germany). Human fibrinogen (SigmaAldrich, St. Louis, MO, USA), collagen (Takeda Pharma, Berlin, Germany), heparin (Ratiopharm, Ulm, Germany), prostacyclin (Calbiochem, Sigma-Aldrich, St. Louis, MO, USA)), and apyrase (Sigma-Aldrich, St. Louis, MO, USA) were purchased.

\subsection{Murine Platelet Preparation and Determination of Cell Count}

Murine platelet preparation followed a previously described protocol $[28,29]$. Blood was drawn from anaesthetized mice via retro-orbital plexus and collected in $300 \mu \mathrm{L}$ heparin $(20 \mathrm{U} / \mathrm{mL})$. The blood was centrifuged at $250 \mathrm{~g}$ at $22^{\circ} \mathrm{C}$ for $5 \mathrm{~min}$. The resulting supernatant was centrifuged at $50 \mathrm{~g}$ for $6 \mathrm{~min}$ to obtain platelet-rich plasma (PRP). PRP was centrifuged at $650 \mathrm{~g}$ using apyrase and $\mathrm{PGI}_{2}$ for $5 \mathrm{~min}$. The remaining pellet was resuspended in Tyrode's buffer (136 mM NaCl, $0.4 \mathrm{mM} \mathrm{Na}_{2} \mathrm{HPO}_{4}, 2.7 \mathrm{mM} \mathrm{KCl}, 12 \mathrm{mM} \mathrm{NaHCO} 3,0.1 \%$ glucose, $0.35 \%$ bovine serum albumin (BSA, Sigma-Aldrich, St. Louis, MO, USA), pH 7.35 , apyrase $(0.02 \mathrm{U} / \mathrm{mL})$ and prostacyclin $(0.5 \mu \mathrm{M}))$ and centrifuged at $650 \mathrm{~g}$ for $5 \mathrm{~min}$. Depending on the following experiment platelets were either resuspended in Tyrode's buffer ( $\mathrm{pH} 7.35)$ with or without $\mathrm{CaCl}_{2}(2 \mathrm{mM})$. Platelets counts and MPV were analyzed by a hematology analyzer (Sysmex, Norderstedt, Germany).

\subsection{Flow Cytometry}

Flow cytometry analysis was performed as described elsewhere [5]. An analysis of murine platelet activation was performed using fluorophore-labeled antibodies for P-selectin expression (Anti-Human/Mouse CD62P APC, Clone Psel.K02.3, eBioscience, San Diego, California, USA, final concentration $20 \mu \mathrm{g} / \mathrm{mL}$ ) and fibrinogen binding (Fibrinogen From Human Plasma, Alexa Fluor ${ }^{\mathrm{TM}} 647$ Conjugate, Life Technologies, Carlsbad, California, USA, final concentration $50 \mu \mathrm{g} / \mathrm{mL}$ ). Heparinized blood was diluted in $500 \mu \mathrm{L}$ Tyrode's buffer ( $\mathrm{pH} 7.35$ ) and washed twice via centrifugation at 650 at $22{ }^{\circ} \mathrm{C}$ for $5 \mathrm{~min}$. 
The remaining pellet was resuspended in $500 \mu \mathrm{L}$ Tyrode's buffer ( $\mathrm{pH}$ 7.35) supplemented with $\mathrm{CaCl}_{2}(2 \mathrm{mM})$. Platelets were stimulated with ADP $(10 \mu \mathrm{M})$, ADP and U46619 $(10 \mu \mathrm{M}$ and $3 \mu \mathrm{M}), \mathrm{CRP}(1$ and $5 \mu \mathrm{g} / \mathrm{mL})$ and thrombin $(0.02$ and $0.01 \mathrm{U} / \mathrm{mL})$ at $22^{\circ} \mathrm{C}$ for $15 \mathrm{~min}$. The reaction was stopped by the addition of PBS (Sigma-Aldrich, St. Louis, MO, USA) and samples were analyzed by use of a FACSCalibur flow cytometer (BD Biosciences, Heidelberg, Germany).

To analyze surface expression of different glycoproteins, blood samples were mixed with the antibodies DyLight649-labeled rat anti-mouse/human integrin $\alpha_{\text {IIb }} \beta_{3}$ (GPIIIa, CD61) (emfret Analytics, Würzburg, Germany, M025-3, no stock concentration provided by emfret Analytics, used dilution 1:10) and DyLight649-labeled anti-mouse GPIb $\alpha$ (Emfret Analytics, Würzburg, Germany, no stock concentration provided by emfret Analytics, used dilution 1:10), and incubated at $22^{\circ} \mathrm{C}$ for 15 min before measurements.

To label the different blood cell types washed whole blood was incubated with APClabeled rat anti-mouse TER-119 (BD Bioscience, Heidelberg, Germany, final concentration $20 \mu \mathrm{g} / \mathrm{mL}$ ), DyLight649-labeled anti-mouse GPIb $\alpha$ (Emfret Analytics, Würzburg, Germany, no stock concentration provided by Emfret Analytics, used dilution 1:10) and APC-labeled rat anti-mouse CD45 (BD, Heidelberg, Germany, final concentration $20 \mu \mathrm{g} / \mathrm{mL}$ ) and analyzed using the Tomato ( $m T$ fluorescence) and the EGFP channel (for $m G$ fluorescence).

\subsection{Fluorescence Microscopy}

For visualization, two drops of peripheral blood were added to $50 \mu \mathrm{L}$ heparin $(20 \mathrm{U} / \mathrm{mL})$. Then one drop was spread on a cover slip and the resulting blood smears were stained with DAPI (Roche Diagnostics, Mannheim, Germany final concentration $1.6 \mu \mathrm{g} / \mathrm{mL}$ ) to stain nuclei of leukocytes. Images were taken with the Zeiss LSM 510-META Confocal laser scanning microscope (Carl Zeiss, Oberkochem, Germany).

Sample slices were prepared from paraffin embedded spleen and femur samples, dewaxed and dehydrated, stained with DAPI (Roche Diagnostics, Mannheim, Germany, final concentration $1.6 \mu \mathrm{g} / \mathrm{mL}$ ) and inundated with FluoromountTM (Sigma-Aldrich, St. Louis, $\mathrm{MO}$, USA). The documentation was done after drying at $4{ }^{\circ} \mathrm{C}$ overnight with microscope Axio Observer.D1 (Carl Zeiss Microscopy GmbH, Oberkochem, Germany, AxioCam MRm, objective LD Plan-Neofluar 40x 0.6 Korr Ph2 M27) and the resulting pictures were analyzed using ImageJ-win64 (Image 1.52, freeware by National Institutes of Health, USA) and ZEN 2.6 (Zen 2.6 blue edition, Carl Zeiss Microscopy GmbH, Oberkochem, Germany).

\subsection{Flow Chamber Experiments to Analyze Thrombus Formation under Flow Conditions}

Coverslips $(24 \times 60 \mathrm{~mm}$ ) were coated with $200 \mu \mathrm{g} / \mathrm{mL}$ fibrillar type I collagen (Takeda Pharma, Berlin, Germany) overnight and then blocked with 1\% BSA solution for at least $60 \mathrm{~min}$. Whole blood samples were perfused through the flow chamber at a shear rate of $1000 \mathrm{~s}^{-1}$ and platelet adhesion and thrombus formation were recorded using the Microscope Axio Observer.D1 (Carl Zeiss Microscopy GmbH, Oberkochem, Germany, AxioCam MRm, objective LD Plan-Neofluar 40x 0.6 Korr Ph2 M27). The images were analyzed for three-dimensional thrombus formation using ZEN 2.6 (blue edition, Carl Zeiss Microscopy $\mathrm{GmbH}$, Oberkochem, Germany).

\subsection{Platelet Adhesion and Spreading}

Experiments were performed as described previously [21,29]. Coverslips $(24 \times 60 \mathrm{~mm})$ were either coated with $100 \mu \mathrm{g} / \mathrm{mL}$ fibrinogen or $200 \mu \mathrm{g} / \mathrm{mL}$ type I collagen at a defined area $(10 \times 10 \mathrm{~mm})$ and incubated overnight at $4{ }^{\circ} \mathrm{C}$. Afterwards the coverslips were blocked with $1 \%$ BSA for $60 \mathrm{~min}$. Then, $2 \times 10^{4}$ isolated platelets were resuspended in $70 \mu \mathrm{L}$ Tyrode's buffer ( $\mathrm{pH} 7.35)$ supplemented with $\mathrm{CaCl}_{2}(2 \mathrm{mM})$, applicated to the prepared coverslips and incubated at room temperature for indicated time points. Nonadherent platelets were carefully removed by rinsing $2 \times$ with PBS. The preparation was fixed by $4 \%$ phosphate buffered formaldehyde at $4{ }^{\circ} \mathrm{C}$ for $10 \mathrm{~min}$, subsequently rinsed again carefully with PBS and inundated with FluoromountTM (Sigma-Aldrich, St. Louis, 
MO, USA). Platelet adhesion was documented after drying at $4{ }^{\circ} \mathrm{C}$ overnight using the microscope Axio Observer.D1 (Carl Zeiss Microscopy GmbH, Oberkochem, Germany, AxioCam MRm, objective LD Plan-Neofluar 40x/0.6 Korr Ph2 M27). The images were analyzed ImageJ-win64 (ImageJ 1.52, freeware by National Institutes of Health, USA) and ZEN 2.6 (Zen 2.6 blue edition, Carl Zeiss Microscopy GmbH, Oberkochem, Germany).

\subsection{Intravital Microscopy of Arterial Thrombosis in Mesenteric Arterioles Following Injury with $\mathrm{FeCl}_{3}$}

Mice (4-5 week of age) were anesthetized with Ketamin (Ketaset, Zoetis, ParsippanyTroy Hills Township, New Jersey, USA, $100 \mathrm{mG} / \mathrm{kg}$ ) and Xylazin (WDT, Garbsen, Germany, $5 \mathrm{mG} / \mathrm{kg}$ ) by intraperitoneal (i.p.) injection. After a midline abdominal incision, the mesentery was exteriorized and arterioles free of fat tissue were injured by topical application of a filter paper saturated with $20 \% \mathrm{FeCl}_{3}$ (Sigma-Aldrich, St. Louis, MO, USA) for $20 \mathrm{~s}$. Thrombus formation was observed with a fluorescence microscope (Axio Observer.D1 Carl Zeiss Microscopy GmbH, Oberkochem, Germany, AxioCam MRm, objective APlan 10x/0.25 Ph1). Time until full occlusion of the vessel (when blood flow had stopped for more than $60 \mathrm{~s}$ ) was measured. Experiments were stopped after $40 \mathrm{~min}$ when no occlusion of the injured vessel was detected.

\subsection{Determination of the Bleeding Time to Investigate Hemostasis in Transgenic Mice}

Mice at the age of 10-12 weeks were anesthetized with Ketamin $(100 \mathrm{mG} / \mathrm{kg})$ and Xylazin $(5 \mathrm{mG} / \mathrm{kg})$ i.p. and placed in prone position. A transverse incision was made with a scalpel at a position where the diameter of the tail is $2.25-2.5 \mathrm{~mm}$ using a gauge to cut a defined size of the tail tip. The tail was immediately immersed in a $15 \mathrm{~mL}$ Falcon tube containing pre-warmed PBS in a water bath to $37^{\circ} \mathrm{C}$ and bleeding was continuously monitored. Each animal was monitored for 20 min even if bleeding stopped in order to detect any re-bleeding events. The time from the incision to the cessation of bleeding was recorded using a stop clock.

\subsection{Generation of Bone Marrow Chimeric Mice}

Twelve-week-old male C57BL/6J mice were exposed to sublethal irradiation of 10 Gray for 3.32 min using the gamma-irradiation device Biobeam GM 2000 (Gamma Service Medical, Leipzig, Germany). Donor bone marrow cells were isolated from femur and tibia of $m T / m G ; P F 4-C r e$ positive mice. After cervical dislocation of the donor animals, the femurs and tibias were freed from the tissue and released from the hip anchorage. Then both hind leg bones were cut off at the distal and proximal ends just behind the joints. Following, the bone marrow cavity was rinsed with chilled PBS through a nylon filter into a reaction tube using a $26 \mathrm{G}$ cannula (Braun, Melsungen, Germany) and centrifuged at $500 \mathrm{~g}$ for $10 \mathrm{~min}$. Lysis of erythrocytes with $1 \mathrm{~mL}$ erythrocyte lysis buffer $\left(155 \mathrm{mM} \mathrm{NH}_{4} \mathrm{Cl}\right.$, $10 \mathrm{mM} \mathrm{KHCO} 3,0.1 \mathrm{mM}$ EDTA; $\mathrm{pH}=7.35$ ) for $5 \mathrm{~min}$ on ice followed. The suspension was centrifuged again at $500 \mathrm{~g}$ for $10 \mathrm{~min}$, the supernatant was removed, and the pellet was resuspended in $150 \mu \mathrm{L}$ PBS and kept on ice until application into recipient animals via the retro-bulbar venous plexus. Bone marrow from one donor hind leg was used per recipient mouse with a minimum of $5 \times 10^{6}$ bone marrow cells. The application volume was set at $150 \mu \mathrm{L}$ of cell suspension. The recipient mice that have been irradiated had the same genotype and sex as the donor mice and were of similar age. After the application of the donor bone marrow, mice were kept under sterile conditions for approximately 2 weeks and supplemented with neomycin through drinking water according to the manufacturer's instructions (Sigma-Aldrich, St. Louis, MO, USA). After a convalescence period of 8 weeks, blood cell counts were determined, and blood cells were analyzed using BD FACSCalibur.

\subsection{Statistical Analysis}

Data are shown as mean \pm standard error of mean (SEM). Statistical significance was analyzed using GraphPad Prism 7 software (San Diego, Californa, USA) and the recommended tests were used as indicated. $p$ values $<0.05$ were considered to be statistically significant. 


\section{Conclusions}

The megakaryocyte/platelet specific double fluorescent Cre reporter mouse $\mathrm{mT} / \mathrm{mG} ; P F 4$ Cre represents an elegant model for lineage tracing, the analysis of cell morphology, transplantation studies, and the differentiation of recombined versus non-recombined platelets. However, a minor fraction of leukocytes showed $m G$ fluorescence in $m T / m G$;PF4-Cre positive mice. Thus, the role of platelets in inflammatory diseases should be assessed carefully and $m G$ fluorescent cells should be examined for their origin.

Author Contributions: Investigation and data analysis: C.F.L., I.K., K.J.K., M.S. and F.R.; writingoriginal draft preparation: M.E.; writing—review and editing: I.K., F.R. and M.E.; supervision: M.E.; project administration and conceptualization: M.E.; funding acquisition: M.E. All authors have read and agreed to the published version of the manuscript.

Funding: This research was funded by grant from the German Research Foundation (Deutsche Forschungsgemeinschaft, DFG), collaborative research center (CRC) 1116 (Project A05), Düsseldorf.

Acknowledgments: We thank. Stefan Gonska, Nina Sarah Gowert and Meike Klier for technical support.

Conflicts of Interest: The authors declare no conflict of interest.

\section{Abbreviations}

$\begin{array}{ll}\mathrm{ADP} & \text { adenosine diphosphophate } \\ \mathrm{BSA} & \text { bovine serum albumine } \\ \mathrm{CaCl}_{2} & \text { calcium chloride } \\ \mathrm{CLEC}-2 & \text { C-type lectin-like receptor } 2 \\ \mathrm{Cre} & \text { cyclization recombination or causes recombination } \\ \mathrm{CRP} & \text { collagen-related peptide } \\ \mathrm{DIC} & \text { differential interference contrast } \\ \mathrm{EDTA} & \text { ethylenediaminetetraacetic acid } \\ \mathrm{FeCl} & \text { iron(III) chloride } \\ \mathrm{GPIb} / \mathrm{VI} & \text { glycoprotein }(\mathrm{GP}) \mathrm{Ib} / \mathrm{VI} \\ \mathrm{HE} & \text { hematoxylin/eosin } \\ \mathrm{KCl} & \text { potassium chloride } \\ \mathrm{KHCO} & \text { potassium bicarbonate } \\ \text { loxP } & \text { locus of X-over P1, recognition site for cre to excision } \\ \mathrm{MFI} & \text { mean fluorescence intensity } \\ \mathrm{mG} & \text { membrane-targeted enhanced green fluorescent protein (EGFP) } \\ \mathrm{min} & \text { minute } \\ \mathrm{mT} & \text { membrane-targeted tandem dimer Tomato (red fluorescent protein) } \\ \mathrm{NaCl} & \text { sodium chloride } \\ \mathrm{NaHCO} & \text { sodium bicarbonate } \\ \mathrm{Na} \mathrm{HPO}_{4} & \text { disodium phosphate } \\ \mathrm{NH} \mathrm{Cl}_{4} \mathrm{Cl} & \text { ammonium chloride } \\ \mathrm{PLD} 1 & \text { phospholipase D1 } \\ \mathrm{PF} 4 & \text { platelet factor } 4 \\ \mathrm{PFA} & \text { paraformaldehyde } \\ \mathrm{PRP} & \text { platelet-rich plasma } \\ \mathrm{rest} & \text { resting } \\ \mathrm{Thr} & \text { thrombin } \\ \mathrm{U} 46 & \text { U46619, thromboxane analogue } \\ \mathrm{vWF} & \text { von Willebrand Factor } \\ & \end{array}$

\section{References}

1. Furie, B.; Furie, B.C. Mechanisms of Thrombus Formation. N. Engl. J. Med. 2008, 359, 938-949. [CrossRef] [PubMed]

2. ISTH Steering Committee for World Thrombosis Day. Thrombosis: A Major Contributor to the Global Disease Burden. J. Thromb. Haemost. 2014, 12, 1580-1590.

3. Mackman, N. Triggers, Targets and Treatments for Thrombosis. Nature 2008, 451, 914-918. [CrossRef] 
4. Gowert, N.S.; Kruger, I. Loss of Reelin protects mice against arterial thrombosis by impairing integrin activation and thrombus formation under high shear conditions. Cell Signal 2017, 40, 210-221. [CrossRef]

5. Elvers, M.; Stegner, D. Impaired Alpha(Ilb)beta(3) Integrin Activation and Shear-dependent Thrombus Formation in Mice Lacking Phospholipase D1. Sci. Signal 2010, 3, ra1. [CrossRef]

6. Miura, Y.; Takahashi, T. Analysis of the Interaction of Platelet Collagen Receptor Glycoprotein VI (GPVI) with Collagen. J. Biol. Chem. 2002, 277, 46197-46204. [CrossRef] [PubMed]

7. Chatterjee, M.; Ehrenberg, A. Molecular Drivers of Platelet Activation: Unraveling Novel Targets for Anti-Thrombotic and Anti-Thrombo-Inflammatory Therapy. Int. J. Mol. Sci. 2020, 21, 7906. [CrossRef]

8. Nieswandt, B.; Watson, S.K. Platelet-collagen Interaction: Is GPVI the Central Receptor? Blood 2003, 102, 449-461. [CrossRef]

9. Krueger, I.; Gremer, L. Reelin Amplifies Glycoprotein VI Activation and AlphaIIb Beta3 Integrin Outside-In Signaling via PLC Gamma 2 and Rho GTPases. Arterioscler. Thromb. Vasc. Biol. 2020, 40, 2391-2403. [CrossRef]

10. Jagadeeswaran, P.; Cooley, B.C. Animal Models of Thrombosis from Zebrafish to Nonhuman Primates. Circ. Res. 2016, 118, 1363-1379. [CrossRef]

11. Thijs, T.; Deckmyn, H. Model Systems of Genetically Modified Platelets. Blood 2012, 119, 1634-1642. [CrossRef]

12. Hart, A.W.; Morgan, J.E. Cardiac Malformations and Midline Skeletal Defects in Mice Lacking Filamin A. Hum. Mol. Gen. 2006, 15, 2457-2467. [CrossRef] [PubMed]

13. Monkley, S.J.; Zhou, X.-H. Disruption of the Talin Gene Arrests Mouse Development at the Gastrulation Stage. Dev. Dyn. 2000, 219, 560-574. [CrossRef]

14. Suzuki-Inoue, K.; Inoue, O. Essential in Vivo Roles of the C-type Lectin Receptor CLEC-2. J Biol Chem 2010, 285, 24494-24507. [CrossRef]

15. Thyagarajan, B.; Guimarães, M.J. Mammalian Genomes Contain Active Recombinase Recognition Sites. Gene 2000, 244, 47-54. [CrossRef]

16. Lepage, A.; Leboeuf, M.n. The $\alpha \mathrm{IIb} \beta 3$ Integrin and GPIb-V-IX Complex Identify Distinct Stages in the Maturation of CD34+cord Blood Cells to Megakaryocytes. Blood 2000, 96, 4169-4177. [CrossRef]

17. Szalai, G.; LaRue, A.C. Molecular Mechanisms of Megakaryopoiesis. Cell. Mol. Life Sci. 2006, 63, 2460-2476. [CrossRef]

18. Falet, H.; Pollitt, A.Y. A Novel Interaction between FlnA and Syk Regulates Platelet ITAM-mediated Receptor Signaling and Function. J. Exp. Med. 2010, 207, 1967-1979. [CrossRef]

19. Tiedt, R.; Schomber, T. PF4-Cre Transgenic Mice Allow the Generation of Lineage-restricted Gene Knockouts for Studying Megakaryocyte and Platelet Function in Vivo. Blood 2007, 109, 1503-1506. [CrossRef]

20. Klatt, C.; Kruger, I. Platelet-RBC Interaction Mediated by FasL-FasR Induces Procoagulant Activity Important for Thrombosis. J. Clin. Investig. 2018, 128, 3906-3925. [CrossRef]

21. Gowert, N.S.; Klier, M. Defective Platelet Activation and Bleeding Complications upon Cholestasis in Mice. Cell Physiol Biochem 2017, 41, 2133-2149. [CrossRef]

22. Muzumdar, M.D.; Tasic, B. A Global Double-fluorescent Cre Reporter Mouse. Genesis 2007, 45, 593-605. [CrossRef]

23. Chudakov, D.M.; Matz, M.V. Fluorescent Proteins and Their Applications in Imaging Living Cells and Tissues. Physiol. Rev. 2010, 90, 1103-1163. [CrossRef]

24. Konig, K. Multiphoton Microscopy in Life Sciences. J Microsc 2000, 200, 83-104. [CrossRef]

25. Calaminus, S.D.J.; Guitart, A.V. Correction: Lineage Tracing of PF4-Cre Marks Hematopoietic Stem Cells and Their Progeny. PLOS ONE 2013, 8. [CrossRef]

26. Pertuy, F.; Aguilar, A. Broader Expression of the Mouse Platelet Factor 4-cre Transgene beyond the Megakaryocyte Lineage. J. Thromb. Haemost. 2015, 13, 115-125. [CrossRef]

27. Rudolph, J.M.; Guttek, K. Characterization of Mice with a Platelet-Specific Deletion of the Adapter Molecule ADAP. Mol. Cell. Biol. 2019, 39, e00365-18. [CrossRef]

28. Donner, L.; Falker, K. Platelets Contribute to Amyloid-beta Aggregation in Cerebral Vessels through Integrin AlphaIIbbeta3induced outside-in Signaling and Clusterin Release. Sci. Signal 2016, 9, ra52. [CrossRef]

29. Elvers, M.; Pozgaj, R. Platelet Hyperreactivity and a Prothrombotic Phenotype in Mice with a Gain-of-function Mutation in Phospholipase Cgamma2. J. Thromb. Haemost. 2010, 8, 1353-1363. [CrossRef] 